\title{
Intervenção Psicoeducativa na Prevenção do Stress na Infância: a Formação de Educadores
}

\section{Psychoeducational Intervention in the Prevention of Childhood stress: the Training of Educators}

\author{
Anabela Sousa Pereira*, Rosa Gomes*, Vanessa Aires**, FelicianoVeiga*** \\ *CIDTFF, Universidade de Aveiro, ** Universidade de Coimbra, *** Instituto de Educação da Universidade de Lisboa, Portugal
}

\begin{abstract}
Resumo
A profissionalidade docente, no quadro dos processos de ensino-aprendizagem, constitui um desafio ao nível da formação especializada, especialmente numa perspetiva life span. Este estudo tem como objetivo geral identificar o tipo de intervenção psicoeducativa que permite atuar ao nível da prevenção do stress. Os resultados mostraram existir correlações moderadas entre as situações indutoras de stress de Causas Internas Psicossociais e as Causas Externas de Componente Familiar. Também as práticas educativas atentas à Cooperação Escola-Família estão correlacionadas com as Atividades de Prevenção das situações indutoras de stress na infância. São recomendados programas de intervenção psicoeducativa na formação de Educadores. Palavras-chave: intervenção psicoeducativa, stress, educação pré-escolar, práticas educativas, formação de professores.
\end{abstract}

\begin{abstract}
Nowadays, the profession of teaching in the panorama of teaching-learning processes is a challenge at the level of specialized training, specially at a life span perspective. This study aimed to evaluate the type of psychoeducational intervention that acts in the prevention of the levels of stress. The results showed moderate correlations between stress inducing situations of Internal Psychosocial Causes and the External Causes of Family Component. Further the educational practices concerned with the Cooperation Family-School are correlated with the Prevention Activities of stress inducing situations in childhood. Psychoeducational intervention programs are recommended in the training of educators.

Keywords: Psychoeducational intervention, stress, preschool education, educational practices, teacher training.
\end{abstract}

As crianças, ao ingressarem no jardim-de-infância, são já confrontadas com várias exigências, quer ao nível das atividades dirigidas e reguladas - no cumprimento de regras -, quer na capacidade de iniciar e manter relacionamentos interpessoais positivos com Educadores e colegas. A qualidade dos contextos, por sua vez, seja ao nível micro, meso, exo, macro e cronossistema (Bronfenbrenner \& Morris, 1998) influenciam o processo de desenvolvimento. Por conseguinte, as práticas educativas devem procurar identificar e interpretar as transições ecológicas que a criança opera, como sujeito dinâmico, em constante interação com o ambiente ecológico.
Compreender a criança é situarmo-nos nos seus micro e meso-sistemas, como a escola e a família, percecionando os outros níveis e apreendendo de cada um dos seus indicadores e referentes, imprescindíveis ao desenho da ação educativa. Segundo a teoria bioecológica do desenvolvimento humano, as interações diárias entre adultos e crianças são processos proximais, que servem como mecanismos primários através dos quais as crianças se desenvolvem (Bronfenbrenner \& Morris, 2006, citado por (Cabell, DeCoster, LoCasale-Crouch, Hamre, \& Pianta, 2013).

As pesquisas publicadas nos últimos 10 anos mostram que os estudos sobre o stress na primeira infância, desenvolvidos em contexto educativo, foram publicados entre 2004 e 2010. Estas pesquisas identificaram como comportamentos indicadores de stress, a rejeição por pares, a ansiedade na separação e as atividades escolares (Murray, \& Harrison, 2005). Colocam também em evidência a formação dos futuros Educadores (Onchwari, 2010), que deverão reforçar as suas competências, de modo a lidarem melhor com o stress dos alunos e a compreenderem o stress em fases precoces, o qual deverá ser continuamente investigado (Vectore, \& Zumstein, 2010). A formação de Educadores/Professores e Psicólogos deverá ser orientada para uma perspetiva de life span do desenvolvimento e à extensão da duração da vida (Jardim, \& Pereira, 2016). A formação superior especializada dos docentes que intervêm no ensino básico e pré-escolar parece ter um papel relevante no desenvolvimento de práticas educativas direcionadas para a prevenção do stress na infância (Gomes, Pereira, \& Aires, 2013).

A revisão da literatura evidenciou também que os estudos sobre o stress na primeira infância, desenvolvidos em contexto familiar, foram publicados entre 2007 e 2012 (Pereira, Gomes, \& Aires, 2013). Na pesquisa de Vallottona, et al. (2012), expõe-se que certos programas como EHS (Early Head Start) têm efeitos positivos na aquisição da linguagem na primeira infância, em situação de stress parental. Por seu lado, Williford, Calkins e Keane (2007) considera que o stress parental está associado a problemas comportamentais externos na criança em idade pré-escolar. Durante a gravidez, o stress familiar pode comprometer o desenvolvimento da criança, especialmente o desenvolvimento cognitivo não-verbal, 
que é particularmente pobre (Henrichs, at al., 2011) nestas situações.

Procurou-se, assim, compreender esta problemática, no sentido de uma maior atuação ao nível da prevenção e da estruturação de uma formação graduada ao nível do $1^{\circ}$ e $2^{\circ}$ ciclos do ensino superior, mas também uma formação contínua, para que estes profissionais possam otimizar estratégias que ajudem a criança a lidar com as situações indutoras de stress.

Um estudo de Onchwari (2010) evidencia que tanto os Educadores como os futuros Educadores consideram estar moderadamente preparados para lidarem com o stress dos seus alunos. $\mathrm{O}$ autor refere ainda que a formação dos futuros Educadores deverá reforçar as suas competências para lidarem melhor com o stress dos alunos. Desenvolver formas de lidar com o stress em contexto educativo, em especial na primeira infância (Gomes, Pereira, \& Aires, 2016), coloca em evidência o papel da formação superior destes profissionais. Para Jardim e Pereira (2016), a implementação de programas de formação ao longo da vida contribui para uma melhoria do desempenho profissional. Também a inserção de abordagens multidisciplinares com "app" (Pereira, Moreira, Chaló, Sancho, Varela, \& Oliveira, 2016) têm sido estudadas ao nível do ensino superior. Estes autores procuraram identificar o tipo de intervenção psicoeducativa que permite atuar ao nível da formação graduada. Este estudo procurou identificar o tipo de intervenção psicoeducativa que permite atuar ao nível da prevenção do stress e refletir sobre as competências pedagógicas dos Educadores.

\section{Método}

Participaram no estudo 294 Educadores do género feminino (98\%) e masculino (2\%), com idades entre os 24 e os 60 anos, a exercer funções em Portugal. A maioria dos Educadores (89\%) é docente em instituições públicas, com 1 a 46 anos de serviço docente. A organização das classes em grupos heterogéneos de idade é a distribuição mais representativa $(82,7 \%)$, distribuídos por classes com crianças de 3,4 e 5 anos (40\%), de 4 e 5 anos (13\%) e 3 , 4,5 e 6 anos (10\%). Os Educadores que participaram no estudo disponibilizam entre 1 a 30 horas por semana, no planeamento da prática pedagógica (PPP).

O instrumento aplicado foi o Protocolo de Prevenção do Stress na Educação Pré-Escolar (P2SEPE), desenvolvido por Gomes e Pereira (2009), no âmbito do doutoramento em psicologia (Gomes, 2012) e validada por Gomes, et al. (2013). A escala usada é do tipo likert, com 4 níveis de resposta, que variam entre zero (nunca) e três (muitas vezes). É um instrumento de avaliação dirigido a
Educadores que desenvolvem atividade docente com crianças em idade pré-escolar (3-6 anos) e que procura identificar competências pedagógicas do Educador para lidar com o stress na infância, avaliar situações que podem ser indutoras de stress e identificar algumas das práticas educativas para prevenção do stress na Infância. Este Protocolo tem as seguintes subescalas: (a) Situações Indutoras de Stress na Infância (ESISI), 18 itens; (b) Práticas Educativas para Lidar com o Stress na Infância (EPELSI), 18 itens; (c) Competências Pedagógicas para Lidar com o Stress na Infância (ECPLSI), 15 itens.

Nos procedimentos, a recolha da amostra decorreu no primeiro trimestre de 2015, junto de Educadores que desenvolvem a atividade docente com crianças dos 3 aos 6 anos, através de questionários de autopreenchimento anónimos e confidenciais. A recolha dos questionários foi obtida através do Google Drive e difundido por correio eletrónico junto dos Diretores de Agrupamento de escolas da região norte e centro de Portugal. As questões éticas foram respeitadas, a participação foi voluntária e cada questionário era acompanhado de informação explicativa dos objetivos, das condições da pesquisa, sendo assegurada a confidencialidade e anonimato dos dados. Utilizámos para a análise dos dados o programa estatístico SPSS, versão 21.0, para MS Windows.

\section{Resultados}

Foram realizadas matrizes de correlação através do coeficiente de correlação de Pearson $(p)$ entre os fatores da ECPLSI, da ESISI e da EPELSI e as variáveis TSD e HPPP, para analisar a validade da convergência. A Tabela 1 mostra os valores das associações obtidas e verifica-se que existem correlações positivas moderadas, significativas a $(\mathrm{p} \leq .01)$, entre a Criatividade Pedagógica e o Planeamento Reflexivo $(r=.519 ; p=.000)$, a Promoção do Bem-estar ( $r=.477 ; p=.000)$, a Formação Graduada $(r=.416 ; p=.000)$, a Cooperação Escola/família $(r=.411$; $p=.000)$ e o Planeamento Psicoeducativo $(r=.345$; $p=.000)$. De igual modo encontramos correlações positivas moderadas entre o Planeamento Reflexivo e as Atividades de Prevenção $(r=.521 ; p=.000), \quad$ a Cooperação Escola/família $(r=.463 ; p=.000)$ e o Planeamento Psicoeducativo $(r=.425 ; p=.000)$. Os resultados mostram ainda existir correlações positivas moderadas entre as situações indutoras de stress de Causas Internas Psicossociais e as Causas Externas de Componente Familiar $(r=.545 ; p=.000)$, as Causas Internas de Componente Familiar $(r=.530 ; p=.000)$ e as Causas Externas de Componente Escolar $(r=.467 ; p=.000)$. 
Tabela 1.

Matriz de correlações entre os fatores que constituem o protocolo P2SEPE e as variáveis TSD e HPPP

\begin{tabular}{|c|c|c|c|c|c|c|c|c|c|c|c|c|}
\hline Fatores & F1 & $\mathrm{F} 2$ & F3 & $\mathrm{F} 4$ & F5 & F6 & F7 & F8 & F9 & F10 & F11 & F12 \\
\hline \multicolumn{13}{|l|}{ ECPLS } \\
\hline I F1 & 1 & & & & & & & & & & & \\
\hline F2 & $.416 * *$ & 1 & & & & & & & & & & \\
\hline F3 & $.171 * *$ & $.147 * *$ & 1 & & & & & & & & & \\
\hline $\mathrm{F} 4$ & $.519 * *$ & $.174 * *$ & - & 1 & & & & & & & & \\
\hline \multicolumn{13}{|l|}{ ESISI } \\
\hline F5 & $.168^{* *}$ & - & $.218 * *$ & $.137 *$ & 1 & & & & & & & \\
\hline F6 & $.242 * *$ & - & $.147 *$ & - & $.545 * *$ & 1 & & & & & & \\
\hline F7 & - & - & $.197 * *$ & - & $.467 * *$ & $.314 * *$ & 1 & & & & & \\
\hline F8 & - & - & $.144 *$ & - & $.530 * *$ & $.314 * *$ & $.295 * *$ & 1 & & & & \\
\hline \multicolumn{13}{|l|}{ EPELS } \\
\hline I F9 & $.477 * *$ & $.284 * *$ & - & $.383^{* *}$ & $.290 * *$ & $.241 * *$ & - & $.197 * *$ & 1 & & & \\
\hline F10 & $.411 * *$ & $.211 * *$ & $.192 * *$ & $.463 * *$ & - & $.161^{* *}$ & - & - & $.465^{* *}$ & 1 & & \\
\hline F11 & $.270 * *$ & - & $\begin{array}{l}- \\
.157 * *\end{array}$ & $.521 * *$ & $.136^{*}$ & - & - & - & $.332 * *$ & $.612 * *$ & 1 & \\
\hline F12 & $.345^{* *}$ & $.156^{* *}$ & - & $.425 * *$ & $.125^{*}$ & $.126^{*}$ & - & - & $.414 * *$ & $.457 * *$ & $.483 * *$ & 1 \\
\hline TSD & $.158^{* *}$ & - & $-.129 *$ & $.139 *$ & - & $.159^{* *}$ & $.132 *$ & - & - & $.289^{* *}$ & $.264 * *$ & $.184 * *$ \\
\hline НРРР & - & - & - & $.162 * *$ & - & - & - & $.125^{*}$ & - & $.174 * *$ & $.187 * *$ & - \\
\hline
\end{tabular}

**Nível de significância de $p \leq .01$

*Nível de significância de $p \leq .05$

Legenda:

F1 - Criatividade pedagógica

F2 - Formação graduada

F3 - Identificação de sintomas

F5 - Causas internas psicossociais

F9 - Promoção do bem-estar
F10 - Cooperação escola/família

F6 - Causas externas de componente familiar

F7 - Causas externas de componente escolar

F4 - Planeamento reflexivo

F8 - Causas internas de componente familiar

F11 - Atividades de prevenção

F12 - Planeamento psicoeducativo

TSD - Tempo de serviço docente

HPPP - Horas de planeamento da prática

pedagógica

Também as práticas educativas atentas à Cooperação Escola Família apresentam correlação positiva moderada com as Atividades de Prevenção $(r=.612 ; p=.000)$ e o Planeamento Psicoeducativo ( $r=.457 ; p=.000)$, de igual modo as Atividades de Prevenção estão correlacionadas com o Planeamento Psicoeducativo $(r=.483 ; p=.000)$.

Pela análise da variância ANOVA também foram encontradas diferenças estatisticamente significativas entre o tempo de serviço docente (TSD) e a maioria dos fatores da EPELSI. Os Educadores com mais tempo de serviço, entre 33 a 46 anos, são os que apresentam médias mais elevadas ao nível das práticas educativas do tipo Cooperação Escola Família $(\mathrm{M}=14,12 ; \mathrm{DP}=0,96 ; \mathrm{F}=$ 9,26; $p=.000)$, das Atividades de Prevenção $(\mathrm{M}=11,81$; $\mathrm{DP}=0,70 ; \mathrm{F}=8,03 ; p=.000)$ e do Planeamento Psicoeducativo $(\mathrm{M}=8,26 ; \mathrm{DP}=0,93 ; \mathrm{F}=5,41 ; p=.001)$. No entanto as práticas educativas para a Promoção do Bem-estar da criança e o TSD não apresentam diferenças estatisticamente significativas $(\mathrm{M}=13,30 ; \mathrm{DP}=2,72 ; \mathrm{F}$ $=0,710 ; p=.547)$. Também os Educadores que mais horas dedicam ao HPPP (entre 11 a 3 horas semanais), são os que apresentam médias mais elevadas ao nível das práticas educativas baseadas em Atividades de Prevenção $(\mathrm{M}=11,87 ; \mathrm{DP}=0,49 ; \mathrm{F}=5,35 ; p=.005)$.

\section{Discussão}

Através dos estudos de correlações e testes paramétricos entre as variáveis estudadas e os fatores, os resultados deste estudo exploratório mostram que a perceção dos Educadores portugueses relativamente às práticas educativas para prevenção do stress está relacionada com as atividades de cooperação entre a escola e a família, sendo os Educadores com mais tempo de serviço, os que mais promovem este tipo de atividades, como por exemplo conhecer a relação da criança com a família ou estabelecer com os pais (ou as novas configurações da instituição familiar) um diálogo regular e construtivo. Também Alcantud, (2015) e Ho e Lin (2015) reconhecem a importância das interações e da cooperação mútua entre pais e professores, no desempenho global das crianças, favorecendo uma educação de infância de qualidade. Por vezes podemos não encontrar dificuldades evidentes ao nível do funcionamento familiar, mas verificam-se um conjunto de condições psicossociais (e.g., desemprego de longa duração; história de abuso de substâncias na família; pobreza; residência em zonas de elevada criminalidade ou violência, etc.) e dúvidas ou dificuldades na gestão da parentalidade, que podem colocar a família em risco, como refere Melo e Alarcão (2009). Poderá, então, ser particularmente benéfico aumentar os fatores de proteção, nomeadamente ao nível da relação pais-filhos, à qual o jardim de infância não poderá ficar indiferente.

Ao nível das competências pedagógicas que os Educadores podem desenvolver para lidar com situações indutoras de stress na infância, destaca-se a criatividade pedagógica, a qual está correlacionada com a formação graduada destes profissionais que, por sua vez, desenvolvem em contexto educativo práticas educativas para a promoção do bem-estar, a cooperação 
escola/família e o planeamento psicoeducativo. Deste modo, o planeamento de atividades que ajudam a criança a lidar com situações adversas (i.e., elevado número de horas no jardim de infância, desenvolvimento de aptidões com as crianças para lidarem com situações difíceis, como seja a morte e o divórcio), são preditivas das práticas educativas que promovem o bem-estar da criança (i.e, (a) ajudar a criança a lidar com situações difíceis; (b) encontrar soluções de apoio aos que rejeitam o sono; (c) desenvolver regularmente atividades de relaxamento) e de práticas psicoeducativas (i.e., (a) desenvolver atividades adequadas ao envolvimento da criança; (b) promover cenários lúdicos para gestão da ansiedade das crianças). Estas competências estão próximas das competências gerais dos Educadores encontradas por Dias (2009), Pereira e Gomes (2012), mas mais específicas para esta problemática. O tempo de planeamento da prática pedagógica revela-se preditivo das atividades de prevenção (i.e., (a) estar atento às crianças que não se envolvem nas atividades de grupo; (b) procurar conhecer os centros de interesse de cada criança; (c) estar atenta às crianças que têm dificuldade em concentrar-se ou de permanecer em determinada atividade).

As dimensões estruturantes das práticas educativas para a sustentabilidade do stress na infância, parecem estar associadas à cooperação escola-família, atividades de prevenção, promoção do bem-estar da criança nas situações indutoras de stress e no planeamento psicoeducativo. Quando identificadas crianças em situações de stress, as práticas educativas podem constituir um fator de proteção ao intervirem nos vários níveis do sistema ecológico - pessoal, familiar e social -, estabelecendo interações positivas (Gomes, Pereira, Veiga, \& Aires, 2015), como seja o caso do apoio prestado pelos colegas e amigos, onde a criança seja valorizada e estimada enquanto membro social (Veiga, \& Fernandes, 2012). O apoio dos pares confere à criança segurança, afeto, sentimentos de pertença e aprovação (Trianes, 2004), possibilitando o ajustamento emocional e, por conseguinte, o desenvolvimento de competências sociais. Outros estudos também enfatizam a qualidade das interações dos Educadores (Cabell, et al., 2013; Williford, Maier, Downer, Pianta, \& Howes, 2013) no envolvimento da criança, tanto nas atividades dirigidas, como no sucesso das suas aprendizagens, mas também nas atividades propostas pela criança (Goble, Hanish, Martin, Eggum-Wilkens, Foster, \& Fabes, 2016).

O papel do Educador como fonte de currículo nas situações de vulnerabilidade e stress (Gomes, 2012; Gomes, \& Pereira, 2007) torna-se essencial, para o desenvolvimento integrado e sustentado da criança, numa perspetiva holística do desenvolvimento. É através de uma ação educativa concertada, que organiza o ambiente e as rotinas, que estabelece um clima de interação social positivo, que encoraja a resolução de problemas e planifica experiências e/ou atividades, alicerçadas nos interesses das crianças, que o Educador potencia a sua intervenção psicoeducativa.

Estes resultados podem ser explicados pela especificidade do planeamento destas atividades, com as competências gerais dos Educadores e ainda com os diferentes estilos de liderança dos Educadores, como mostra o estudo de Ho e Lin (2015), ao criarem uma atmosfera envolvente que promova um ambiente de empatia e bem-estar.

Melhorar a qualidade da formação de Professores/ Educadores e incentivar o seu desempenho profissional também é destacado por Cadório e Simão (2013). Dotar estes profissionais com competências que lhe confiram qualidades para observar, planificar, organizar e avaliar o ambiente educativo, promover o desenvolvimento e aprendizagem da criança, de modo a lidar com as situações indutoras de stress, constitui um dos desafios atuais da formação de Educadores e outros agentes educativos, que participam em contextos diferenciados, como resultado da globalização ou por integração em ONG,s (Aires, 2017), que participam em ambientes multiculturais.

As implicações deste estudo evidenciam a necessidade de adequar a formação de Educadores que desenvolvem a atividade docente ou equiparada, bem como a formação contínua, numa perspetiva life-span, para que estes profissionais possam otimizar estratégias que ajudem a criança a lidar com as situações de stress, sendo necessários, no entanto, estudos confirmatórios.

\section{Referências}

Aires, V. (2017). Do tecto do mundo vê-se o recreio: a infância nepalesa (em)revista. In J. P. Gaspar \& E. Santos (Coords.), Acolhimento Juvenil no Mundo: Respostas Sociais e Estratégias Terapêuticas fundadas na Cultura (pp.181-200). Lisboa: Edições Ex-Libris.

Alcantud, P. (2015). Grupos interactivos en educación infantil: Primer paso para el éxito educativo. Intangible Capital, 11(3), 316-332. http://dx.doi.org/10.3926/ic.658

Bronfenbrenner, U., Morris, P.A. (1998). The ecology of developmental processes. In: Damon, W.; Lerner, R. M. (Orgs.). Handbook of child psychology, Vol. 1: Theoretical models of human development. New York: John Wiley, (pp. 993-1028).

Cabell, S.Q., DeCoster, J., LoCasale-Crouch, J., Hamre, B.K., \& Pianta, R.C. (2013). Variation in the effectiveness of instructional interactions across preschool classroom settings and learning activities. Early Childhood Research Quarterly, 28(4), 820-830. http://dx.doi.org/10.1016/j.ecresq.2013.07.007

Cadório, L., \& Simão, A. (2013). Mudanças nas Concepções e Práticas dos Professores. Lisboa: Edições Vieira da Silva.

Dias, I. (2009). Promoção de competências em educação. INDEA. Instituto Politécnico de Leiria.

Goble, P., Hanish, L.D., Martin, C. L., Eggum-Wilkens, N. D., Foster, S. A., \& Fabes, R.A. (2016). Preschool Contexts and Teacher Interactions: Relations with School Readiness. Early education and development. 27(5), 623-641. http://dx.doi.org/10.1080/10409289.2016.1111674

Gomes, R., \& Pereira, A. (2007). Perspectivas dos Educadores sobre as Situações Indutoras de Stress: estudo exploratório em contextos educativos para a 
infância. Revista Psicologia e Educação. VI, 2, Dez, 61-72. Universidade da Beira Interior: Covilhã.

Gomes, R. (2012). (Re)configuração das práticas educativas na prevenção do stress na infância. Tese de Doutoramento, Universidade de Aveiro, Aveiro, Portugal. http://hdl.handle.net/10773/12707.

Gomes, R., Pereira, A., \& Aires, V. (2013). Práticas educativas para lidar com o stress na infância (EPELSI): estudo psicométrico. In (Orgs.) Anabela Pereira, Manuela Calheiros, Paula Vagos, Inês Direito, Sara Monteiro, Carlos F. Silva \& Ana Gomes. Atas VIII Simpósio Nacional de Investigação em Psicologia, (pp. 227-233). Aveiro: Universidade de Aveiro.

Gomes, R., Pereira, A., Veiga, F., \& Aires, V. (2015). Prevention Practice of Stress in Preschool Education: Educators' Perception. International Journal of Case Studies, 4(12), 15-19. Retrived from http://www.casestudiesjournal.com/volume-4-issue$12 /$

Gomes, R., Pereira, A. \& Aires, V., (2016). Prevenção do stress na infância: Contributo para a sua avaliação com o P2SEPE. In I. Leal, C. Godinho, S. Marques, P. Vitória \& J. L. Pais Ribeiro (Org.), Actas do $11^{\circ}$ Congresso Nacional de Psicologia da Saúde (pp. 659666). Lisboa: Sociedade Portuguesa de Psicologia da Saúde.

Henrichs, J.; Schenk, J.J., Kok, R., Ftitache, B., Schmidt, H.G., Hofman, A., Jaddoe, V.W.V., Verhulst, F.C., \& Tiemeier, H. (2011). Parental Family Stress during Pregnancy and Cognitive Functioning in Early Childhood: The Generation R Study. Early Childhood Research Quarterly. 26(3), 332-343. http://dx.doi.org/10.1016/j.ecresq.2011.01.003.

Melo, A. T., \& Alarcão, M. (2009). Centros de Apoio Familiar e Aconselhamento Parental: proposta de um modelo global de organização. Psicologia \& Sociedade, 21(1), 55-64. https://dx.doi.org/10.1590/S010271822009000100007 .

Murray, E., \& Harrison, L.J. (2005). Children's Perspectives on Their First Year of School: Introducing a New Pictorial Measure of School Stress. European Early Childhood Education Research Journal. 13(1), 111-127. http://dx.doi.org/10.1080/13502930585209591.

Onchwari, J. (2010). Early Childhood Inservice and Preservice Teachers' Perceived Levels of Preparedness to Handle Stress in Their Students. Early Childhood Education Journal. 37(5), 391-400. http://dx.doi.org/10.1007/s10643-009-0361-9.

Pereira, A., \& Gomes, R.M. (2012). Avaliação de competências pedagógicas dos educadores e o stresse na infância. INFAD: Revista de Psicologia. Infancia y Adolescencia em un mundo en crisis y cambio. 1(1), 2936.

Pereira, A., Gomes, R., \& Aires, V. (2013). O stress na infância em contexto educativo: meta análise reflexiva. In Chaleta, M., Grácio, M., Saraiva, M., Vieira, I. et al (Coords.). Actas da II International Conference "Learning and Teaching in Higher Education" and "Learning Orchestration in Higher Education, (pp. 92106). Évora: Universidade de Évora.
Pereira, A., \& Jardim, J. (2016). Perceived impact of lifelong training in teachers. Revista Interacções. 12(42), 22-31.

Pereira, A. S., Moreira, A. A., Chaló, P., Sancho, L., Varela, A., \& Oliveira, C. (2016). Development Challenges of a Full Integrated App in Higher Education. In L. Briz-Ponce, J. Juanes-Méndez, \& F. García-Peñalvo (Eds.), Handbook of Research on Mobile Devices and Applications in Higher Education Settings (pp. 1-24). Hershey, PA: IGI Global. https://dx.doi.org/10.4018/978-1-5225-0256-2.ch001

Trianes, M. V. (2004). O Stress na Infância: prevenção e tratamento. Porto: Edições ASA

Vallotton, C.D., Harewood, T., Ayoub, C.A., Pan, B., Mastergeorge, A.M., \& Brophy-Herb, H. (2012). Buffering boys and boosting girls: The protective and promotive effects of Early Head Start for children's expressive language in the context of parenting stress. Early Childhood Research Quarterly. 27(4), 695-707. http://dx.doi.org/10.1016/j.ecresq.2011.03.001

Vectore, C., \& Zumstein, L.S. (2010). Utilização de recursos mediacionais para a identificação de estressores em pré-escolares. Psicologia Escolar Educacional. $\quad 14(2)$,

https://dx.doi.org/10.1590/S141385572010000200013 .

Veiga, F. H., \& Fernandes, A. (2012). Autorregulação em crianças do jardim-de-infância: adaptação da escala Teacher Ratings of Behavioral Self-regulation in Preschool Children. In D. Domingues et al. (eds.). Atas do $12^{\circ}$ Colóquio de Psicologia, Educação, Aprendizagem e Desenvolvimento: Olhares Contemporâneos através da Investigação, (pp. 536544). Lisboa: ISPA. http://hdl.handle.net/10400.12/5410

Williford, A.P., Calkins, S.D., \& Keane, S.P. (2007). Predicting Change in Parenting Stress across Early Childhood: Child and Maternal Factors. Journal of Abnormal Child Psychology. 35(2), 251-263. http://dx.doi.org/10.1007/s10802-006-9082-3.

Williford, A.P., Maier, M.F., Downer, J.T., Pianta, R.C., \& Howes, C. (2013). Understanding how children's engagement and teachers' interactions combine to predict school readiness. Journal of Applied Developmental Psychology. 34(6), 299-309, https://doi.org/10.1016/j.appdev.2013.05.002.

\section{Agradecimentos}

Os autores agradecem a participação dos Educadores neste estudo.

Este trabalho é financiado por Fundos Nacionais através da FCT - Fundação para a Ciência e a Tecnologia, I.P., no âmbito do projeto UID/CED/00194/2013.

\section{Declaração de conflito de interesse}

Os autores declaram que não há conflitos de interesse. 